\title{
Identification of potential Zinc deficiency responsive genes and regulatory pathways in rice genotypes by Weighted Gene Co-expression Network Analysis
}

\section{Blaise Pascal Muvunyi}

Chinese Academy of Agricultural Sciences Agricultural Genomes Institute at Shenzhen

\section{Xiang Lu}

Chinese Academy of Agricultural Sciences Agricultural Genomes Institute at Shenzhen

\section{Sang He}

Chinese Academy of Agricultural Sciences Agricultural Genomes Institute at Shenzhen

\section{Guoyou Ye (D G.Ye@irri.org )}

Chinese Academy of Agricultural Sciences Agricultural Genomes Institute at Shenzhen https://orcid.org/0000-0002-9943-4815

\section{Research Article}

Keywords: Zinc deficiency, Weighted Gene Coexpression Analysis, Biofortification, Rice

Posted Date: April 26th, 2021

DOl: https://doi.org/10.21203/rs.3.rs-442740/v1

License: (c) (1) This work is licensed under a Creative Commons Attribution 4.0 International License. Read Full License 


\section{Abstract}

\section{Background}

Zinc ( $\mathrm{Zn})$ malnutrition has been linked to serious health concerns in humans. Targeting genetic biofortification of rice grain $\mathrm{Zn}$ can efficiently improve the global $\mathrm{Zn}$ nutritional status. To genetically enhance rice grain $\mathrm{Zn}$ content, the genetic and molecular mechanisms of $\mathrm{Zn}$ deficiency response need to be elucidated. Here, Differential Gene Expression Analysis (DGEA) and Weighted Gene Coexpression Analysis (WGCNA) were established to identify modules of coexpressed genes, the most preserved genes and molecular pathways regulating $\mathrm{Zn}$ deficiency response in rice varieties.

\section{Results}

Twelve modules of coexpressed genes were obtained by WGCNA using 1649 differentially expressed genes (DEGs) from our DGEA and $2121 \mathrm{Zn}$ genes from earlier studies. Three modules (TTA-M, TRA-M and CA-M) were judged the most relevant for $\mathrm{Zn}$ deficiency based on their richness in the well-recognized $\mathrm{Zn}$ deficiency responsive (ZDR) genes and molecular pathways. 96 (17\%), 188 (47.6\%) and 96 (24\%) genes from TTA-M, TRA-M and CA-M modules respectively, were significantly expressed in the DGEA. These coexpressed DEGs (CoDEGs) were considered as the most preserved $Z n$ deficiency responsive genes. Of the well-known ZDR genes, only OsZIP8, OsZIP10, OsMT1a and OsNAAT1 were preserved. Functional annotations for all CoDEGs from the identified ZDR modules showed that glutathione metabolism and biosynthesis of secondary metabolites were the most quickly upregulated molecular pathways. Lastly, a biology-informed gene-gene interaction network analysis (GGIN) indicated that CoDEGs including OsLSI3, OsWOX11, OsNRT1.1B, OsPSK5, OsSWN6 and OsMID1 strongly interact with the recognized ZDR genes in the ZDR modules. Curiously, these CoDEGs were previously validated for other economic traits in rice.

\section{Conclusion}

Findings from this study provide comprehensive insights into the molecular mechanisms of Zn deficiency response in rice and may facilitate gene and pathway prioritization, to enhance $Z n$ use efficiency (ZUE) and $\mathrm{Zn}$ biofortification in rice.

\section{Background}

Zinc malnutrition is affecting about 1.2 billion of people and has been associated with detrimental health concerns including children stunting, weak immune system and brain development (Black, 1998; Dardenne, 2002). Regarding the current pandemic of COVID-19, Zn malnutrition will exacerbate infection rates (Akhtar et al.; Huizar et al., 2020; Van Der Straeten et al., 2020), underlining the need for Zn biofortification.

Fortifying $\mathrm{Zn}$ in rice, a staple crop for nearly 3.5 billion of people, can significantly benefit the global $\mathrm{Zn}$ nutritional status (Van Der Straeten et al., 2020). Agronomic approaches to enhance grain Zn content 
through chemical fertilization for example, are not only unecomomical, but also ineffective, as Zn tends form insoluble complexes which limits its uptake from plant root tissues (Alloway, 2008). Favorably, natural genetic variations in rice germplasm are sufficient to attain $\mathrm{Zn}$ concentrations requirements of 28 ppm in the polished rice grain (Van Der Straeten et al., 2020). Zn efficient (ZE) genotypes have relatively higher ZUE and perform better than Zn inefficient (ZI) genotypes under low Zn soils or nutrient solutions (Nanda et al., 2017). Also, ZE genotypes exhibit higher growth of crown roots (Nanda and Wissuwa, 2016a) and greater rates of the exudation of low-molecular weight organic acids (LMWOA) metalchelators, such as nicotianamine synthase (NAs), mugineic acid (MAs) or 2'-deoxymugenic acid (DMAs) from the phytosiderophore family (Ishimaru et al., 2011; Ptashnyk et al., 2011; Widodo et al., 2010). Such traits have been linked to the loading of both Zn or Fe in rice grains (Banakar et al., 2017; Masuda et al., 2012). In addition to these LMWOAs, Zinc transporter genes mainly ZIP (zinc-regulated transporters and iron-regulated transporter proteins), HMAs (heavy metal ATPases) and MTPs (metal tolerance proteins) are essential for the uptake, distribution, and redistribution of $Z n$ across different plant tissues under low Zn deficiency conditions (Ishimaru et al., 2005; Olsen and Palmgren, 2014). Further, transcription factor (TF) genes such as OsNAC, OsIRO2, and OsIRO3 are also known to regulate the expressions of several $\mathrm{Zn}$ or Fe responsive genes (Banerjee and Chandel, 2011; Ogo et al., 2006). As to molecular pathways affected by Zn deficiency, previous transcriptomic studies have consistently shown that Zn deficiency responsive (ZDR) genes significantly enrich Zinc transmebrane activity, transmebrane transport activity, phenylpropanoid biosynthesis and glutathione metabolism functional gene sets (Bandyopadhyay et al., 2017; Nanda et al., 2017; Zeng et al., 2019b). Nevertheless, the most conserved ZDR genes and molecular pathways are not known. Also, the interaction networks involving the known (experimentally validated) ZDR genes are yet to be investigated.

Weighted gene co-expression network analysis (Langfelder and Horvath, 2008) is a systems biology approach that leverages on the extent of co-expression between genes to define their connectivity. Subsequently, it allows studying the universal network nature of a transcriptome (Zhao et al., 2010). For example, integrating transcriptomic analysis and WGCNA in rice enabled to find the global Cadmium (Cd)-regulated DEGs (Tan et al., 2017). Also, WGCNA was useful to functionally annotate the genome of rice (Childs et al., 2011) and construct the atlas of maize by integrating different omics data (Walley et al., 2016). In a transcriptome co-expression network, genes are represented by nodes which are linked together by edges based on the weighted co-expression across samples. The most connected genes within a network are assembled as modules, while the highly connected genes within a module are referred to hubs (Zhang and Horvath, 2005). Co-expression network architecture is inherent of cellular organization, with the functional modules build up from several interacting molecules (Hartwell et al., 1999).

Here, DGEA and WGCNA were implemented to identify (1) functional modules of coexpressed genes in Zn-stressed rice genotypes (2) coexpressed DEGs (CoDEGs) and their preserved molecular functions, and (3) genes showing strong connectivity with the experimentally validated (herein referred to well-known or recognized) ZDR genes. Subsequently, modules of coexpressed genes were constructed by combining DEGs from our own samples including ZI (IR26 and IR64) and AUS (WCP22 and KALBOR026) genotypes, 
and the other publicly reported ZDR genes. The significance of a module for Zn deficiency response was dictated by the recognized ZDR functional annotations enriched by genes coexpressing in that module. Genes from the identified ZDR modules which are DEGs in our DGEA were judged as the most preserved, and so were their respective functional annotations. These functionally annotated CoDEGs are good candidates to track functional markers which can be incorporated into breeding schemes to improve rice grain Zn content. Lastly, gene-gene interaction network (GGIN) or protein-protein interaction network (PPIN) of the ZDR modules identified genes or proteins with strong connectivity with the well-recognized ZDR genes. We speculate that these identified hub genes could also be involved in the mechanistic of $\mathrm{Zn}$ stress response as their linked well-known ZDR genes.

\section{Results}

\section{Identification of differentially expressed genes.}

Gene expression analysis was performed for root and shoot tissues of two ZI (IR26 and IR64) and AUS (UCP122 and KALBOR026) rice genotypes using DESeq2 R package (Love et al., 2014). To obtain sufficient DEGs for the next stage of WGCNA, two approaches were used. A condition-based gene expression analysis (CEA) where gene expression for each ZI or AUS genotype was studied under Zn deficiency condition (Zn supply vs Zn deficiency), and a genotype-based expression analysis (GEA) by setting ZI genotypes as reference level for each of AUS genotype (IR26/IR64 vs UCP122/KALBOR026). Overall, 1649 unique genes (Table 1 and Additional file1-10: Table S1-10) were differentially expressed (FDR $<0.01, \log _{2}$ fold $>=2$ or $<2$ ). 1179 of the 1649 DEGs were novel ZDR genes, while 470 DEGs have been reported in previous studies (Fig. 1a and Additional file 11: Table S11). Under CEA, the well-known ZDR genes such as ZIP (OsZIP1, OsZIP5, OsZIP10 and OsZIP8), a phytosiderophores family gene (OsNAAT1), and a metallothionein gene (OsMT1a) were induced by Zn deficiency treatment in ZI or AUS genotypes. Under low Zn supply conditions and GEA, the expressions ZIP genes and phytosiderophores family genes (OSNAAT1 and OsNAS3) were significantly higher in the root sample of AUS genotypes than in the same tissue of IR26 genotype (Additional file 9-10: Table S9-10).

\section{Functional annotation of differentially expressed genes.}

Functional annotations of DEGs (under CEA) by STRING tool version:11 (Mering et al., 2003) indicated that Zinc ion transmembrane transporter activity GO term was significantly enriched by DEGs upregulated by Zn deficiency treatment in both ZI and AUS genotypes. However, significant differences between UCP122 and other genotypes were detected in the enrichment of the DNA-binding transcription factor activity GO term. The latter was significantly upregulated in the root tissue of UCP122 yet downregulated in the same tissue of KALBOR026 and IR26 genotypes. Similarly, Zinc ion transmembrane transporter activity GO term was upregulated in root tissues of UCP122, while for all the other genotypes, it was only upregulated in crown tissues. 
Table 1

Number of DEGs obtained under CEA and GEA in AUS and ZI genotypes.

\begin{tabular}{|c|c|c|c|c|c|}
\hline \multirow[t]{2}{*}{ Contrast levels } & & \multirow[t]{2}{*}{ Contrast categories } & \multicolumn{3}{|c|}{ Expressed genes $($ FDR $<0.01)$} \\
\hline & & & $\log _{2}$ fold $>=2$ & $\log _{2}$ fold $<2$ & Total \\
\hline \multirow{10}{*}{$\begin{array}{l}\text { Zn supply vs Zn Def } \\
\text { (CEA) }\end{array}$} & & IR26_root & 93 & 19 & 112 \\
\hline & ZI & IR26_crown & 14 & 56 & 70 \\
\hline & & IR64_root & 20 & 24 & 44 \\
\hline & & IR64_crown & 35 & 276 & 311 \\
\hline & & Total non-redundant genes & 145 & 312 & 457 \\
\hline & & UCP122_root & 129 & 11 & 140 \\
\hline & AUS & UCP122_crown & 4 & 10 & 14 \\
\hline & & KALBOR026_root & 49 & 94 & 143 \\
\hline & & KALBOR026_crown & 36 & 8 & 44 \\
\hline & & Total non-redundant genes & 203 & 87 & 290 \\
\hline \multirow{15}{*}{$\begin{array}{l}\text { ZI vs AUS genotypes } \\
\text { (GEA) }\end{array}$} & & Under Zn deficiency & & & \\
\hline & & IR26 vs UCP122_crown & 97 & 178 & 275 \\
\hline & & IR26 vs UCP122_root & 155 & 387 & 526 \\
\hline & & IR26 vs KALBOR026 root & 137 & 398 & 535 \\
\hline & & IR26 vs KALBOR026 crown & 27 & 55 & 82 \\
\hline & & IR64 vs UCP122_crown & 98 & 83 & 181 \\
\hline & & IR64 vs UCP122_root & 46 & 123 & 169 \\
\hline & & IR64 vs KALBOR026 root & 54 & 205 & 259 \\
\hline & & IR64 vs KALBOR026 crown & 115 & 94 & 209 \\
\hline & & Total non-redundant genes & 497 & 912 & 1419 \\
\hline & & Under Zn supply & & & \\
\hline & & IR26 vs UCP122_crown & 23 & 37 & 60 \\
\hline & & IR26 vs UCP122_root & 58 & 119 & 177 \\
\hline & & IR26 vs KALBOR026 root & 49 & 80 & 129 \\
\hline & & IR26 vs KALBOR026 crown & 11 & 31 & 42 \\
\hline
\end{tabular}




\begin{tabular}{|lllll|}
\hline Contrast levels & Contrast categories & \multicolumn{2}{l|}{ Expressed genes (FDR < 0.01) } \\
\hline IR64 vs UCP122_crown & 25 & 60 & 85 \\
\hline IR64 vs UCP122_root & 27 & 157 & 184 \\
\hline IR64 vs KALBOR026 root & 26 & 73 & 99 \\
\hline IR64 vs KALBOR026 crown & 11 & 51 & 66 \\
\hline Total unique genes & 129 & 294 & 413 \\
\hline
\end{tabular}

Also, cellulose metabolic process was downregulated in crown tissues of all the genotypes but was not enriched by DEGs from the UCP122 genotype. These findings infer that UCP122 cultivar may have unique phenotype under low Zn supply conditions. All the significantly enriched functional gene sets are provided (Table 2 and Additional file 12-15: Table S12-15). 
Table 2

Functional annotations of DEGs in ZI and AUS genotypes after Zn deficiency treatment (under CEA).

\begin{tabular}{|c|c|c|c|}
\hline \multirow[t]{2}{*}{ Samples } & Go term/ & \multirow[t]{2}{*}{ GO term/pathway description } & \multirow[t]{2}{*}{ FDR } \\
\hline & $\begin{array}{l}\text { KEGG } \\
\text { pathway_ID }\end{array}$ & & \\
\hline \multirow[t]{8}{*}{ ROOT_KALBOR026 } & G0:0015250 & Water channel activity & +0.00087 \\
\hline & GO:0015318 & $\begin{array}{l}\text { Inorganic molecular entity transmembrane } \\
\text { transporter activity }\end{array}$ & ${ }^{+} 0.00087$ \\
\hline & G0:0015075 & Ion transmembrane transporter activity & ${ }^{+} 0.0199$ \\
\hline & map00480 & Glutathione metabolism & ${ }^{+} 0.0282$ \\
\hline & map00940 & Phenylpropanoid biosynthesis & ${ }^{+} 0.0378$ \\
\hline & Go:0140110 & Transcription regulator activity & *0.00018 \\
\hline & GO:0003700 & DNA-binding transcription factor activity & *0.00065 \\
\hline & G0:0044249 & Cellular biosynthetic process & $\star 0.0012$ \\
\hline \multirow[t]{6}{*}{ CROWN_KALBOR026 } & GO:0005385 & Zinc ion transmembrane transporter activity & $\begin{array}{l}+3.45 \mathrm{E}- \\
05\end{array}$ \\
\hline & G0:0010333 & Terpene synthase activity & $\begin{array}{l}+3.45 \mathrm{E}- \\
05\end{array}$ \\
\hline & GO:0022890 & $\begin{array}{l}\text { Inorganic cation transmembrane transporter } \\
\text { activity }\end{array}$ & ${ }^{+} 0.00011$ \\
\hline & map00904 & Diterpenoid biosynthesis & ${ }^{+} 0.0036$ \\
\hline & GO:0030243 & Cellulose metabolic process & *0.0052 \\
\hline & GO:0071555 & Cell wall organization & *0.0067 \\
\hline \multirow[t]{5}{*}{ ROOT_UCP122 } & G0:0005488 & Binding & ${ }^{+} 0.00019$ \\
\hline & G0:0003700 & DNA-binding transcription factor activity & +0.0045 \\
\hline & G0:0003824 & Catalytic activity & +0.0045 \\
\hline & G0:0005385 & Zinc ion transmembrane transporter activity & +0.0134 \\
\hline & GO:0030244 & Cellulose biosynthetic process & +0.038 \\
\hline
\end{tabular}

+ : GO terms/KEGG pathways significantly $(F D R<0.05)$ enriched by upregulated genes.

*: GO terms/KEGG pathways significantly $(F D R<0.05)$ enriched by downregulated genes. 
KEGG

pathway_ID

CROWN_UCP122

ROOT_IR26

\section{NO significant annotations found}

G0:0050667 Homocysteine metabolic process

$+0.0057$

Go:0008652 Cellular amino acid biosynthetic process $\quad{ }^{+} 0.031$

G0:0009086 Methionine biosynthetic process $\quad{ }^{+} 0.031$

map00940 Phenylpropanoid biosynthesis $\quad+0.0044$

map01110 Biosynthesis of secondary metabolites $\quad{ }^{+} 0.0044$

map00130 Ubiquinone and other terpenoid-quinone $\quad{ }^{+} 0.0298$ biosynthesis

map00360 Phenylalanine metabolism

$+0.0298$

GO:0005488 Binding

$\star 0.0278$

GO:0046872 Metal ion binding

$\star 0.0278$

GO:0003700 DNA-binding transcription factor activity

$\star 0.0371$

CROWN_IR26

G0:0005385 Zinc ion transmembrane transporter activity

$+1.62 \mathrm{E}-$

06

G0:0006073 Cellular glucan metabolic process

$\star 1.70 \mathrm{E}-$

08

G0:0030243 Cellulose metabolic process

$\star 1.70 \mathrm{E}-$

08

G0:0071555 Cell wall organization

$\star 1.26 \mathrm{E}-$

07

${ }^{+} 0.0142$

G0:0046872 Metal ion binding

$+0.006$

map00940 Phenylpropanoid biosynthesis

$\star 0.003$

G0:0034219 Carbohydrate transmembrane transport

*0.0026

GO:0051119 Sugar transmembrane transporter activity

G0:0005385 Zinc ion transmembrane transporter activity
$+1.62 \mathrm{E}-$ 06

+ : GO terms/KEGG pathways significantly $(F D R<0.05)$ enriched by upregulated genes.

*: GO terms/KEGG pathways significantly $(F D R<0.05)$ enriched by downregulated genes. 


\begin{tabular}{|llll|}
\hline Samples & $\begin{array}{l}\text { Go term/ } \\
\text { KEGG } \\
\text { pathway_ID }\end{array}$ & GO term/pathway description & FDR \\
\hline G0:0006073 & Cellular glucan metabolic process & *1.70E- \\
& & \\
\hline G0:0030243 & Cellulose metabolic process & *1.70E- \\
& G0:0071555 & Cell wall organization & *1.26E- \\
\hline & & 07 \\
\hline$+:$ GO terms/KEGG pathways significantly (FDR<0.05) enriched by upregulated genes. & \\
\hline *: GO terms/KEGG pathways significantly (FDR<0.05) enriched by downregulated genes. & \\
\hline
\end{tabular}

Construction of the global network of coexpressed modules of genes.

To construct the global network of coexpressed genes, raw counts from nine Zn-deprived samples were obtained from https://www.ebi.ac.uk/ena, then processed and merged with eight Zn-deprived samples from our own study (Fig. 1b and Additional file 17: Table S17). Samples from public datasets included ZE (Nipponbare, IR55179 and RIL46) and ZI (KP, IR64, IR74) genotypes. The selected genes for WGCNA were 1649 DEGs from this project and $2121 \mathrm{Zn}$ responsive genes reported from seven previous studies (Fig. 1a and Additional file 11: Table S11).

We set the module minimum size and the threshold to merge correlated modules at 16 and 0.6 , respectively. The merged raw counts from different projects were corrected for batch effects, tissue types and plant growth stage -mediated covariances. The expression variance was also stabilized as indicated in WGCNA tutorial

https://horvath.genetics.ucla.edu/html/CoexpressionNetwork/Rpackages/WGCNA/faq.html. These parameters resulted into 2076 genes which coexpressed across 13 different modules (Table 3), including a module of unassigned genes. All the modules were relabeled with short abbreviations for reference.

\section{Functional annotations of the identified modules.}

To associate modules to Zn deficiency responsiveness, GO terms and KEGG pathways enrichment by genes within each module were studied using STRING version:11 (http://string-db.org/) (Mering et al., 2003). Apart from genes from P-M module, all the other modular genes significantly enriched at a minimum one GO term or KEGG pathway (Table 3). Intriguingly, molecular functions and genes recognized for $\mathrm{Zn}$ uptake, distribution, and grain $\mathrm{Zn} / \mathrm{Fe}$ content functions in rice, preferably coexpressed in TTA-M, TRA-M and CA-M modules (Fig. 2a). Therefore, all the subsequent characterizations were implemented on these putative ZDR modules. 
Table 3

Functional annotations of coexpressed modules of genes.

\section{Module's colour | Assigned Module preserved GO terms | preserved KEGG pathways labell Number of genes in a module \\ (Bonferroni $p$-value $<0.05$ )}

Blue |TTA-M|555 *

Transmembane transport activity ; Zinc ion transmembane transport ; iron ion homeostasis, etc. I Metabolic pathways; Photosynthesis, etc.

Midnightblue|TRA-M|395 *

Transcription regulator activity; Zinc ion transmembrane transporter activity, etc. I Biosynthesis of secondary metabolites; alpha-Linolenic acid metabolism.

Greenyellow|CA-M|393 *

Grey60 |AP-M|145

Royalblue |PB-M|217

Lightcyan|CG-M|65

Darkturquoise|PHEN-M|96

Darkgreen|PHOT-M|41

Darkgrey|CA-M|35

Black|RES-M|122

Pink|DR-M|395

Purple|P-M|79

Grey|FAT-M|94
Catalytic activity; metal ion binding, etc. I Metabolic pathways;

Phenylpropanoid biosynthesis, Glutathione metabolism, etc.
No significant GO terms I phenylpropanoid biosynthesis; fatty acid metabolism, etc.

Cell growth I glyoxylate and dicarboxylate metabolism; cutin, suberine and wax biosynthesis; biosynthesis of unsaturated fatty acids.

No significant GO annotations | Phenylpropanoid biosynthesis; phagosome; stilbenoid, diarylheptanoid and gingerol biosynthesis.

Photosynthesis | Cyanoamino acid metabolism.

Starch and sucrose metabolism; glycine, serine and threonine metabolism.

Cold acclimation; photosynthesis; response to abiotic stress | porphyrin and chlorophyll metabolism.

Response to external stimulus; response to extracellular stimulus | Amino sugar and nucleotide sugar metabolism, etc.

No significant GO annotation found I DNA replication, alpha-Linolenic acid metabolism, plant-pathogen interaction.

No significant GO annotations | No significant KEGG annotations found.

No significant GO annotation found | Fatty acid elongation.

* Zn deficiency responsive modules (ZDR modules).

$\mathrm{Zn}$ deficiency responsive modules are putative regulators of $\mathrm{Zn}$ deficiency response in rice.

Many of the well-known ZDR genes and their molecular pathways were found in ZDR modules. Genes coexpressing in the TTA-M module included for example, Zinc ion transmembrane transporters (OsZIP3, OsZIP8, OsHMA2 and OsHMA3), a MAs transporter (OsZIFL4/OsTOM1), transmembrane transporters (OSYSL 16, OsYSL 18 and OSMTP4), metal-NA complexes transporters (OsYSL4 and YSL6), metal binding (OSMT4IC, OsMT1B and OSMT1a) and iron homeostasis genes (OsSHR1 and OsNRAMP6). Equally, 
functional annotations including Zinc ion transmembrane transporter activity (OsMTP1 and OsZIP2), organic substance (OsMCM4, OSMPK7, OsCIPK9, OsCESA6, OSCSLF6, etc.), oxidoreductase activity (OsCKX5, OsDAO, genes from CYP family genes, etc.), transmembrane transporter activity (OsNRAMP6, OsMST6, OSABCG44, etc.) and transcription regulator activity (OSIRO2, OSERF67, OsONAC12, OsSWN6, OSDREB1C, OsJAZ TFs genes, etc.) were detected the TRA module. CA-M was another important module, with catalytic activity as its most enriched GO term. Genes including OsZIP1O and MAs biosynthesis genes (OsSAMS1, OsSAMS2, OsNAAT1 and OsDMAS1 coexpressed in the CA-M module. Collectively, these findings are suggestive of potential key roles of ZDR modules in the regulating $Z n$ deficiency molecular mechanisms in rice. All the genes coexpressed across ZDR modules and their function annotations are provided (Additional file 18-23: Table S18-23).

\section{Preserved $\mathrm{Zn}$ deficiency responsive genes and their molecular functions.}

To find the most preserved genes, we intersected ZDR module genes with DEGs from the DGEA (under CEA). 96 (17\%), 188 (47.6\%) and 96 (24\%) genes from TTA-M, TRA-M and CA-M modules respectively, were responsive to $\mathrm{Zn}$ deficiency in our DGEA (Fig. $2 \mathrm{~b}$ and Additional file 24-28: Table S24-28). Among all the well-known ZDR genes, the most preserved were Zinc transmembrane transport genes OsZIP8 (TTA-M) and OsZIP10 (CA-M) which were significantly upregulated in crown tissues of all ZI genotypes and KALBOR026 genotype, a metallothionein gene OsMT1a (TTA-M) which was upregulated in the root tissue of UCP122 genotype, and a phytosiderophores family gene OsNAAT1 (CA-M) which upregulated in root and crown tissues of all AUS genotypes and IR64 genotypes (Additional file 29-30:Table S29-30). Enrichment analysis of all coexpressed DEGs (CoDEGs) showed that genes which upregulated in the root tissue of UCP122 genotype affected the highest number of functional sets vs genes from all the other sample genotypes.

Of the 140 genes upregulated in root tissue of UCP122 genotype (Table 1), 88 genes coexpressed in TRA module, and significantly enriched $77 \mathrm{GO}$ terms including DNA-binding transcription factor activity, cellulose biosynthetic processes, catalytic activity, response to stimuli, etc (Fig. 2c and Additional file 34:

Table S34). Contrary, in the same module, five of the GO terms upregulated in the root sample of UCP122 genotype (including DNA-binding transcription factor activity, binding, sequence specific DNA binding, etc.) were rather downregulated in the root tissue of KALBOR26 genotype. This was consistent with our findings with the DGEA that suggested possible phenotypic differences between UCP122 and other varieties during $\mathrm{Zn}$ deficiency conditions. On the other hand, another set of CoDEGs from the TTA-M module upregulated the glutathione metabolism in root tissues of both AUS genotype. The other preserved and upregulated functional gene sets included the secondary metabolites biosynthesis (CA-M module) and transmembrane transport (TTA-M module) which were significantly enriched by CoDEGs from the root sample of IR26 and KALBOR026 genotypes, respectively (Fig. 2c).

\section{Identification of hub genes in $\mathrm{Zn}$ deficiency responsive modules.}

The Maximal Clique Centrality (MCC) algorithm (Chin et al., 2014) was applied to identify hub genes across ZDR modules. The first 30 hub genes as ranked by their scores from the strongest to weakest, are 
provided (Additional file 35-37: Table S35-37). Among the well-known ZDR genes, only OsZIP3 and OsYLS18 ( both from TTA-M module) featured in the top 30 hub genes. To identify the most Zn deficiency relevant hub genes, we rather adopted a biology-informed approach where CoDEGs directly connected with at least three recognized ZDR genes, or proteins directly interacting with at least one well-known ZDR protein were considered as ZDR specific hub genes or proteins (Additional file 38: Fig. S1-2). The wellknown ZDR genes or proteins for which the interactions were searched against were: OsZIP10, OsNAAT1, OsSAMS1, OsSAM2, OsDMAS1 and OsYSL 10 (CA-M module); OsIRO2, OsZIP2, and OsNRAMP6 (TRA-M module); OsZIP3, OsZIP8, OsMT1a, OsHMA2, OsHMA3, OsYSL6, OsYSL 14,OsYSL 18, OsNRAMP4, OsNRAMP7 and OsTOM1 for the TTA-M module. Genes meeting the set criteria included OsbHLH120, OsWOX11, OsPSK5, OsNAC121, OsPGIP1, OsMID1, OsMYB55, OsJAmyb and OsMPH1 (CA-M module), OsSWN6, ONAC12, OsERF67, OsbHLH108 (TRA-M module), and OsHMA1, OsLIS3, OsGSTU12, OsNRT1.1B, OsMTI4C, OsMT1g, OsbZIP79 and OsTRX for TTA-M module (Fig. 3a-c and Additional file 18-20: Table S18-20).

Curiously, the https://funricegenes.github.io/ search database for functionally validated genes in rice, showed that several of the CoDEGs (Additional file 29-31: Table S29-31) linking up the recognized ZDR genes have established agronomic functions such as transport of essential nutrients, biotic and abiotic stress tolerance (Table 4 and Fig. 3a-c). 
Table 4

Experimentally validated genes which are hub genes for the known ZDR genes.

$\begin{array}{lll}\text { Module Gene name MSU_ID } & \begin{array}{l}\text { Gene functions (retrieved from } \\ \text { https://funricegenes.github.io/) }\end{array}\end{array}$

TTA-M OsNRT1.1B* LOC_Os10g40600 Key regulator of Nitrogen use efficiency in rice.

Enhances of Selenium concentration in rice grains.

OsLSI3* LOC_Os10g39980 Essential for Silicon distribution in vascular structures of rice.

OsMT1g* LOC_Os12g38290 Confers multiple abiotic stress tolerance in rice.

OsRNS4* LOC_Os11g05480 Improves salinity stress tolerance.

OsTRX LOC_Os12g08730 Enhances carotenoid and chlorophyll content.

CA-M OsBHL120 LOC_Os09g28210 A QTL for root thickness and lengths in upland rice.

OsWOX11* LOC_Os07g48560 Enhances crown root development.

Enhances drought stress tolerance.

Improves potassium deficiency tolerance.

Regulates cytokinin signaling pathway.

OsMYB55 $\quad$ LOC_Os05g48010 Regulates amino acid metabolism.

Enhances heat stress tolerance and immunity.

Regulates hormonal signaling. $\mathrm{OsMPH}^{+} \quad$ LOC_OsO6g45890 $\begin{aligned} & \text { Modulates plant height and enhances drought stress } \\ & \text { tolerance. }\end{aligned}$

Enhances Cadmium stress tolerance.

OsMID1*+ LOC_Os05g37060 Improves drought stress tolerance

Negatively regulates Arsenic stress tolerance

OSPSK5* LOC_Os12g05260 Target of osa-miR168a regulating seed vigor

OsPGIP1* LOC_Os05g01380 Enhances sheath blight tolerance

TRA-M OsSWN6* LOC_Os04g45340 Positively regulates the secondary wall biosynthetic. processes.

*: Coexpressed in ZDR modules and expressed in our DGEA (CoDEGs).

+: R2R3-type MYB transcription factor gene.

\section{Discussion}


This study indicates that integrating WGCNA and prior biological knowledge on ZUE trait is an effective method to add value on previous findings from GWAS, qtl mapping, transgenic, miRNA and gene transcriptomic studies on $\mathrm{Zn}$. This apporach enabled to establish modules of coregulated genes, determining conserved molecular functions, and predicting new putative $\mathrm{Zn}$ reponsive genes which may facilitate the improvement of Zn use efficiency and Zn biofortification in rice.

\section{The significance of Zinc deficiency responsive modules.}

Our results indicated that molecular pathways and genes known to play essential roles in $\mathrm{Zn}$ homeostasis and $\mathrm{Zn}$ loading into rice grain coexpressed in ZDR modules. These genes included for instance, ZIP genes (OsZIP2, OsZIP3, OsZIP10 and OsZIP8), heavy metal ATPase genes (OsHMA1, OsHMA2 and OsHMA3), mugeneic acid (MAs) or nicatiamine synthase (NAs) genes (OsNAAT1, OsTOM1, OsDMAS1, OsSAMS1, and OsSAM2), metallothionein (MT) protein coding gene (OsMT1a) and Zn/Fe transcription regulator genes (OSIRO2 and OsIRO3). OsZIP8 gene is involved in $\mathrm{Zn}$ uptake and transport in rice (Lee et al., 2010). And the expression of OsZIP10 gene correlated with high iron (Lee et al., 2009) and $\mathrm{Zn}$ (Maurya et al., 2018) concentrations in rice seeds. Apart from ZIP genes, OsHMA2 and OsHMA3 have also been experimentally validated. OsHMA2 is a long-distance transporter of $\mathrm{Zn}$ and $\mathrm{Cd}$ (Yamaji et al., 2013), while OsHMA3 enhances Cd stress tolerance and the expression of other $\mathrm{Zn}$ transporter genes (Cai et al., 2019; Sasaki et al., 2014).

MAs and NAs are metal chelators from the phytosiderophores family genes and play essential roles in the accumulation Zn/Fe into grain rice, while mitigating the loading of $\mathrm{Cd}$ (Banakar et al., 2017). Earlier studies demonstrated that OsTOM1, a DMA efflux transporter gene, enhanced tolerance to both Zn (Ishimaru et al., 2011) and Fe (Nozoye et al., 2011) deficiencies in rice. Further, targeting MAs genes was proposed as a single strategy to improve both $\mathrm{Zn} / \mathrm{Fe}$ in rice endosperm (Singh et al., 2017). MTs genes are also metal chelators that bind transition metals such as Zn (Sinclair and Krämer, 2012). Transgenic rice plants overxpressing OsMT1a showed enhanced expressions of Zn-induced CCCH zinc-finger TFs, ROS scavenging capacity, drought stress tolerance and Zn concentrations in rice tissues (Yang et al., 2009).

Coexpression of all these $\mathrm{Zn}$ transporters, phytosiderophores family genes and metal chelators genes across TTA-M, TRA-M and CA-M modules suggests relevant functions of ZDR modules in the molecular mechanism $\mathrm{Zn}$ deficiency response.

\section{Conserved genes and molecular pathways across $\mathrm{Zn}$ deficiency responsive modules.}

By intersecting ZDR modules genes and DEGs from our study we obtained the most conserved transcriptionally active genes. The most significantly enriched functional annotations by these CoDEGs were glutathione metabolism, DNA binding transcription factor, transmembrane transport, and biosynthesis of secondary metabolites including phenylpropanoid and phenylalanine metabolism. Interestingly, these conserved molecular pathways were only enriched by genes expressed in root tissues, underscoring the crucial role of root tissue in Zn deficiency regulation (Fig. 2c). Glutathione (GSH; Y- 
glutamyl-cysteinyl-glycine) is a low- molecular-weight (LMW) intracellular signalling molecule with indispensable antioxidant and abiotic stress tolerance roles (Noctor et al., 2012). The significant enrichment of glutathione metabolism by $\mathrm{Zn}$ stress induced genes was also reported by previous studies in rice (Bandyopadhyay et al., 2017; Zeng et al., 2019a). Zeng et al. (2019a) showed that Zn supply repressed the activity involved in glutathione metabolism genes, suggesting that glutathione has essential roles in Zn stress response. Further, it was shown that the antioxidant roles of GSH enhanced Cd stress tolerance in rice (Chen et al., 2010). In this study, a set of DEGs that upregulated in root tissues of both AUS genotypes and coexpressed in the TTA-M module, significantly enriched the glutathione metabolism pathway. An earlier study indicated that salt stress tolerant rice cultivar Pokkali had significantly greater levels of GSH compared to a sensitive variety, IR64 (El-Shabrawi et al., 2010). Whether the exudation of GSH or the expression of GSH pathway genes is linked to higher ZUE in ZE genotypes is worthy further investigations, for example, through transgenic or crop modelling approaches.

Conversely, secondary metabolites KEGG pathways such as phenlypropanoid biosynthesis was preserved in a group CoDEGs from CA-M module which were upregulated in the root sample of IR26 genotype. The upregulation of phenlypropanoid biosynthesis pathways in rice tissues under low $\mathrm{Zn}$ supply was also reported in previous studies (Nanda et al., 2017; Zeng et al., 2019a). Phenylpropanoids constitute the major group of secondary metabolites in plants (Sharma et al., 2019). Phenylpropanoids can chelate metal ions to enhance the mobilization and uptake of essential elements suh as $\mathrm{Zn}$, iron, manganese, potassium, calcium and magnesium (Seneviratne and Jayasinghearachchi, 2003). The high conservation of both glutathione and phenlypropanoid biosynthesis pathways in root tissues of AUS and ZI is another evidence for the vital roles of LMW root effluxes in enhancing Zn uptake from low Zn environments.

\section{Closest hub genes of the well-known ZDR genes are known stress responsive genes.}

Genes with strong connectivity with the validated ZDR genes were identified. Several of these genes or their homologuous were shown to play major roles in different morphogenetic events, biotic and abiotic stress responses by previous investigators. These included for example, a TF gene OsbHLH120 which interacted with validated ZDR genes in the CA-M module. OsbHLH120 is a QTL for root growth and thickness, and improves drought tolerance in upland rice (Li et al., 2015). The homologous gene of OsbHLH120, OsIRO2/OsbHLH56, is a well-known TF gene that positively regulates the expression of several genes involved into Fe uptake in rice (Ogo et al., 2009; Ogo et al., 2007). Both TFs contain an homeodomain Leucine Zipper (HD- Zip 1) element known to mediate of hormonal responses and response to stimuli (Ariel et al., 2007). In contrast, another homologous gene, OsIRO3, negatively regulates Fe uptake (Wang et al., 2020; Zheng et al., 2010) but interacted with OsDMAS1 in the PPIN (Additional file 38: Fig. S2c). OsIRO3 has a similar binding site with OsIRO2 (5'-CACGTGG-3'). Whether the activity of OsbHLH120 under $\mathrm{Zn} / \mathrm{Fe}$ deficiency is similar to OsIRO2 or OsIRO3 requires further studies. Unknown targets of OsbHLH120/ OsIRO3 may contribute to Fe/Zn hypersensitivity. 
OsWOX11 was an other important hub gene for known ZDR genes. OsWOX11 is a regulator of cytokinin signalling pathway, crown and root hair development, and enhances drought stress tolerance (Cheng et al., 2016; Neogy et al., 2019). An ealier study associated crown development with Zn stress tolerance in ZE genotypes (Nanda and Wissuwa, 2016b). In rice, cytokinin inhibits crown root development, while auxin promotes that process (Debi et al., 2005; Kitomi et al., 2011). Here, a cytokining gene (OsCKX5) from the ZDR modules was downregulated by Zn deficiency in root sample of KALBOR026 genotype (Additional file 31: Table S31), constistent with the significant higher root biomass observed in KALBOR026 genotype relative to ZI genotypes (IR26 and IR64) (Lu et al., 2020). However, OsWOX11 and its homologous gene OsWOX6 only upregulated in root samples of a ZI genotype (IR26) (Additional file 31: Table S31) with less root biomass relative to AUS genotypes (Lu et al., 2020). OsWOX11 may have essential roles in Zn stress response, as its neigbour genes. However, more investigations are needed to illuminate the regulation of ctyokinin signalling by OsWOX11 during Zn deficiency conditions.

On the other hand, in the TTA-M, OsNRT1.1B gene coexpressed and interacted with known ZDR genes such as OsZIP3, OsHMA2 and OsYSL 18 (Fig. 3). OsNRT1.1B is a QTL for nitrate use efficiency divergence in rice cultivars (Hu et al., 2015) and enhances nitrogen uptake under low nitrogen conditions in cultivated rice (Fan et al., 2016). Also, OsNRT1.1B improves Selenium levels in rice grains (Zhang et al., 2019). A previous study indicated that OsNRT1.1B was induced by Zn stress conditions (Bandyopadhyay et al., 2017). Here, the expression of OsNRT1.1B was significantly repressed in root tissues of a Zn sensitive genotype, IR64 (Additional file 29: Table S29). As a transporter gene, OsNRT1.1B could also have significant functions in the translocation of $\mathrm{Zn}$ in different rice tissues.

Still in the TTA-M module, OsLSI3 linked up OsHMA3, OsHMA2, OsYSL14 and OsYSL18. OsLSI3 and its homologue OSLIS2 involve in the distribution and redistribution of Arsenic across vascular structures in rice (Yamaji et al., 2015). OsLSI2, is a silicon efflux transporter responsible for arsenic accumulation in rice grain (Ma et al., 2008). However, exogenous Silicon is known to enhance gas exchange capacity in rice plants under Zn stress (Song et al., 2014), and the uptake of essential micronutrients (Zn and Manganese) and macronutrients (Phosphorous, potassium, Calcium and Magnesium) in rice and other monocots plants under heavy metal stress conditions (Keller et al., 2015; Tripathi et al., 2013). On the other side, Silicon also reduced Zn uptake in root tissues of maize and cotton plants (Bokor et al., 2015). Here, OsLIS1, OsLIS2 and OsLSI3 were significanlty upregulated in root samples of KALBOR026 relative to IR26 in the same tissue under Zn stress conditions (Additional file 10: Table S10). OsLIS2 was also significantly induced by $\mathrm{Zn}$ stress relative to $\mathrm{Zn}$ supply conditions in root tissues of KALBOR026 genotype. It is likely that OsLIS2 / OSLIS3 have similar functions as their linked Zn/cd transporter genes. However, their specific roles in $\mathrm{Zn}$ homeostasis remain to be elucidated.

Another gene connecting the well-known ZDR genes was OsHMA1. It is presumed that OsHMA1 involves in $\mathrm{Zn}$ stress response or detoxification of $\mathrm{Zn}$ excess like its orthologue AtHMA1 (Takahashi et al., 2012). OsHMA1 transcripts accumulated by more than 7 folds under Zn-stress condition relative to Zn supply conditions (Suzuki et al., 2012). 
In this study, OsHMA1 was significantly upregulated in crown tissues of AUS (KALBOR026) and ZI (IR64) genotypes (Additional file 29: Table S29). This suggests that OsHMA1 could also have similar functions as its homoguous genes OsHMA2 or OsHMA3.

Laslty, many of the validated R2R3-type MYBTFs genes (Katiyar et al., 2012) linked up several known ZDR genes across the ZDR modules (Fig. $3 \mathrm{C}$ and Table 4). These MYB TF genes were also reported in previous transcriptomic studies on Zn deficiency in rice, but none these has been experimentally validated for $Z n$ deficiency functions. Considering that coexpressing and interacting genes are likely to share same functions, the identified hub genes which have been validated for the other economic traits in rice could also have key roles in enhancing Zn deficiency tolerance as their linked well-known ZDR genes. Thus, targeting these putative novel ZDR genes may benefit the concurrent improvement of several agronomic traits in rice.

\section{Conclusions}

A comprehensive elucidation of functional pathways within the framework of co-expression network enabled to identify 3 key modules of coexpressed genes under $Z n$ deficiency, 380 global conserved genes and their pathways, and key hub genes in the interaction networks of $Z n$ responsive genes. Of the wellrecognized Zn defiency responsive genes, only OsZIP8, OsZIP10, OsMT1a and OsNAAT1 were expressed in our samples and conserved in the identified key modules. OsZIP3 and OsYSL 18 were the only recognized $Z n$ responsive genes detected among the top hub genes in the key modules. Several other hub genes with strong interactions with the recognized Zinc defiency responsive genes were found. Results from this investigation may for instance facilitate genomic selection breeding schemes in targeting relevant molecular pathways and genes to enhance ZUE and Zn biofortification in rice.

\section{Materials And Methods}

A comprehensive pipeline used from DGEA to hub genes identification is provided (Additional file 38: Fig. S3).

\section{Sample description and data acquisition for DEG analysis.}

Procedures from sample preparation and Zn deficiency treatment to the generation of the Fragment Per Kilobase Millions (FPKM) data frame were previously performed in our laboratory, and are as described in a published study (Lu et al., 2020). Briefly, samples consisting of one-week old root or crown tissues of two ZI (IR26 and IR64) and two AUS (UCP122 and KALBOR026) genotypes were supplied (control) or deprived of $Z n$ nutrient regime for one month (Zn stress treatment). The FPKM table as generated by our biological collaborators consisted of 36 columns covering the four genotypes, two tissues (root and crown) per each genotype, two replicates per tissue of each genotype and two treatment conditions ( $\mathrm{Zn}$ supply or Zn deficiency). Two replicates per tissue for each genotype were treated as one independent biological sample under Zn supply or Zn deficiency conditions (Additional file 16-17: Table S16-17). 
Differential gene expression analysis (DGEA) was only carried out for samples from our laboratory using the Bioconductor R package DEseq2 (Love et al., 2014). Genes were considered differentially expressed if passing the stringent criterions of fold-change $\geq 2$ or $\leq-2$, q_value $\leq 0.01$, and false discovery rateadjusted $p$ value $($ FDR $)<0.01$.

\section{Sample description, RNAseq data acquisition and processing for WGCNA}

The global gene co-expression network was built using the Weighted Gene Coexpression analysis (Langfelder and Horvath, 2008) with 17 independent biological samples obtained after collapsing related replicates by their averages (Additional file 17: Table S17). Eight of the 17 samples were Zn-stressed samples from this study (as described in the section above), six others (IR55179, RIL46, Nipponbare, IR64, IR74 and KP) were from a study by (Nanda et al., 2017), and the remaining were from (Zeng et al., 2019b). Overall, nine genotype samples (IR55179, RIL46, IR64, IR74, IR26, NIPPONBARE, KP, UCP122 and KALBOR026) were used for WGCNA. These samples were representative of different varietal ecotypes including Japonica (NIPPONBARE and KP), Indica (IR55179, RIL46, IR64, IR74 and IR26) and AUS (UCP122 and KALBOR026), and different Zn use efficiency (ZU) levels. Only raw counts from Zn-stressed samples were retrieved from NCBI data repository and fed into RNAseq pipeline.

The RNAseq pipeline first consisted of adapter removal and low-quality reads trimming by Trimmomatic tool (Bolger et al., 2014). Next, clean reads were mapped to the MSU7 rice reference genome using HISAT alignment tool (Anders et al., 2015). The annotation and reference genome files (MSU7) were obtained from Illumina's iGenomes project

(support.illumina.com/sequencing/sequencing_software/igenome.html). The poorly aligned reads were filtered out using the filter SAM tool version 0.1.19 (Li et al., 2009). The SAM tool generated a BAM file of quality reads which was fed into the subread tool (Liao et al., 2014) along with gene model annotation file. The resulting gene count matrix was converted to FPKM values and merged with the Zn deficiency FPKM table provided by our biological collaborators. All the used samples were pair-end sequenced libraries.

\section{Data quality processing for WGCNA}

The FPKM values from the above steps were rounded to their nearest integers and standardized using the rlog() function of DESeq2 $R$ package. Rows with minimum counts less than 10 were also filtered out. As the only variation of interest was genotypic variation, effects from other factors such as tissue type, development stage, Zn stress treatment duration were corrected using removeBatchEffect() of limma $\mathrm{R}$ package. Samples' PCA and clustering before and after controlling the unwanted variations are as illustrated in (Additional file 38: Fig. S4a-b). The resulting normalized FPKM values were finally filtered with their MAD (median and median absolute deviation) to reduce noises by removing low-expressed or non-varying genes (Langfelder and Horvath, 2008).

\section{Gene co-expression analysis and module construction}


Only Zn deficiency responsive gene loci comprising of genes reported in 7 previous studies using different methods (e.g., GWAS, qtl mapping, miRNA, transgenic and transcriptomic studies) and DEGs from this study were selected for network construction (Additional file 11: Table S11). WGCNA (version 1.49) $R$ package was used to assemble modules of coexpressed genes (Langfelder and Horvath, 2008). The scale free topology was used for choosing the softpower threshold $(\beta)$ for the computation of adjacency matrix as $a_{i j}=\left|s_{i j}\right|^{\beta}$; where $s_{i j}$ is the correlation between gene $i$ and gene $j$ (Zhang et al., 2005). Soft thresholding refers to reducing low correlation continuously by powering the correlation between genes to that threshold using $\beta$ parameter. The pickSoftThreshold() function was used to determine the $\beta$ value. The soft power threshold of 13 was selected as the first power that surpasses the scale-free topology fit index of 0.85 (Additional file 38: Fig. S5a) (Ramírez-González et al., 2018). Next, a topological overlap matrix (TOM) was constructed from adjacency matrices using $\beta$ value of 13 (Additional file 38: Fig. S5b). TOM enables to determine the network connectivity of a gene as defined by all its adjacencies

with the rest of genes for network generation. Step by step procedure was finally established to construct a consensus network with parameters: $\operatorname{minModuleSize}=16$; dynamicMods $=$ cutreeDynamic $($ dendro $=$ geneTree, distM = dissTOM, deepSplit = 2, pamRespectsDendro = FALSE, minClusterSize = minModuleSize). After generation of the dynamic tree modules, modules were labeled to colors and merged with mergeCloseModules() function.

\section{Network visualization and hub genes identification}

Gene-gene and protein-protein network structures from ZDR modules were represented graphically using Cytoscape (v3.8) (Cline et al., 2007). Genes or proteins directly connected with at least three well-known ZDR genes or proteins were retained for a finer gene-gene interaction network (GGIN) visualization. The top hub genes in each ZDR modules were based on the Maximal Clique Centrality method implemented via the cytoHubba plugin (Chin et al., 2014) in Cytoscape (v3.8) software.

\section{Identification of transcription regulators, transcription factors, protein kinases genes}

Transcription factors were identified using the Plant Transcription Factor Database (PlantTFDB version 5.0 (http://planttfdb.cbi.pku.edu.cn/index.php) (Pérez-Rodríguez et al., 2010). Transcription regulators and protein kinases were predicted using iTAK version 1.6 software

(http://itak.feilab.net/cgi-bin/itak/index.cgi) (Zheng et al., 2016).

\section{Functional annotation enrichment analysis}

Gene Ontology (GO) terms and Kyoto Encyclopedia of Genes and Genomes (KEGG) pathways affected by the DEGs from this study, coexpressed genes across all the modules, and the differentially coexpressed genes across ZDR modules were identified using STRING tool version:11 (http://stringdb.org/) (Mering et al., 2003) at FDR $<0.05$. 


\section{List Of Abbreviations}

ZUE: Zinc Use Efficiency

DGEA: Differential Gene Expression Analysis

ZDR : Zn Deficiency Responsive

ZE : Zn Efficient

ZI : Zn Inefficient

WGCNA: Weighted Gene Coexpression Analysis

DEGs : Differentially Expressed Genes

CoDEGs : Coexpressed Differentially Expressed Genes

MCC: Maximal Clique Centrality

CEA: Condition-based gene Expression Analysis

GEA : Genotype-based gene Expression Analysis

LMWOA : Low-Molecular Weight Organic Acids

GGIN : Gene-Gene Interaction Network

PPIN : Protein-Protein Interaction Network

NAs : Nicotianamine synthase

MAs : Mugineic Acid

DMAs : Deoxymugenic Acids

ZIP : Zinc-regulated transporters and Iron-regulated transporter Proteins

HMAs : Heavy Metal ATPases

MTPs : Metal Tolerance Proteins

ROS : Reactive Oxygen Species

MT : Metallothionein

TF : Transcription factor 
TFR : Transcription factor regulator

\section{Declarations}

\section{Ethics approval and consent to participate}

Not applicable

\section{Consent for publication}

Not applicable

\section{Availability of data and material}

All datasets generated for this study are included in the article/supplementary material, further inquiries can be directed to the first author.

\section{Competing interests}

The authors declare that they have no competing interests.

\section{Funding}

This work was supported by the Agricultural Science and Technology Innovation Program, National Key R\&D Program of China (2020YFE0202300) and Shenzhen Science and Technology Projects (JCYJ20200109150650397).

\section{Author's contributions}

Gouyou Ye conceived the project. Xiang Lu and Blaise Pascal MUVUNYI prepared the samples and collected data. Blaise Pascal MUVUNYI and Sang He analyzed the data. Blaise Pascal MUVUNYI drafted the manuscript. Sang He and Gouyou Ye revised the manuscript. All the authors have read, edited and approved the current version of the manuscript.

\section{Acknowledgements}

The authors wish to thank Dr.Liu Chen for revising the manuscript.

\section{References}

Akhtar S, Das JK, Ismail T, Wahid M, Saeed W, Bhutta ZA (2021) Nutritional perspectives for the prevention and mitigation of COVID-19. Nutr Rev 79(3):289-300 https://doi.org/10.1093/nutrit/nuaa063 
Alloway BJ (2008) Zinc in soils and crop nutrition.

http://www.topsoils.co.nz/wp-content/uploads/2014/09/Zinc-in-Soils-and-Crop-Nutrition-Brian-J.Alloway.pdf .Accessed 15 Apr 2021

Anders S, PyI PT, Huber W(2015) HTSeq-a Python framework to work with high-throughput sequencing data. Bioinformatics 31(2):166-169

https://doi.org/10.1093/bioinformatics/btu638

Ariel FD, Manavella PA, Dezar, CA, Chan RL (2007) The true story of the HD-Zip family. Trends in plant science (12)9:419-426 https://doi.org/10.1016/j.tplants.2007.08.003

Banakar R, Alvarez FA, Díaz-Benito P, Abadia J, Capell T, Christou P (2017) Phytosiderophores determine thresholds for iron and zinc accumulation in biofortified rice endosperm while inhibiting the accumulation of cadmium. Journal of Experimental Botany 68(17):4983-4995 https://doi.org/10.1093/jxb/erx304

Bandyopadhyay T, Mehra P, Hairat S, Giri J (2017) Morpho-physiological and transcriptome profiling reveal novel zinc deficiency-responsive genes in rice. Fun \& Int Gen (17):565-581 https://doi.org/10.1007/s10142-017-0556-x

Banerjee S, Chandel G (2011a) Understanding the role of metal homeostasis related candidate genes in $\mathrm{Fe} / \mathrm{Zn}$ uptake, transport and redistribution in rice using semi-quantitative RT-PCR. Plant Mol Biol Biotechnol 2:33-46

Black MM (1998) Zinc deficiency and child development. The American journal of clinical nutrition (68):464S-469S https://dx.doi.org/10.1093\%2Fajcn\%2F68.2.464S

Bokor B, Bokorová S, Ondoš S, Švubová R, Lukačová Z, Hýblová M, Szemes T, Lux A (2015) lonome and expression level of Si transporter genes (Lsi1, Lsi2, and Lsi6) affected by Zn and Si interaction in maize. Environmental Science, Research Pollution 22(9):6800-6811 https://doi.org/10.1007/s11356-014-3876-6

Bolger AM, Lohse M, Usadel B (2014) Trimmomatic: a flexible trimmer for Illumina sequence data. Bioinformatics 30(15):2114-2120 https://doi.org/10.1093/bioinformatics/btu170

Cai H, Huang S, Che J, Yamaji N, Ma JF (2019) The tonoplast-localized transporter OsHMA3 plays an important role in maintaining $\mathrm{Zn}$ homeostasis in rice. Journal of experimental botany 70(10):2717-2725 https://doi.org/10.1093/jxb/erl054

Chen F, Wang F, Wu F, Mao W, Zhang G, Zhou M (2010) Modulation of exogenous glutathione in antioxidant defense system against $\mathrm{Cd}$ stress in the two barley genotypes differing in Cd tolerance. Plant Physiology, Biochemistry 48(8):663-672 https://doi.org/10.1016/j.plaphy.2010.05.001 
Cheng S, Zhou DX, Zhao Y (2016) WUSCHEL-related homeobox gene WOX11 increases rice drought resistance by controlling root hair formation and root system development. Plant Sign \& Beh11(2):e1130198. https://doi.org/10.1080/15592324.2015.1130198

Childs KL, Davidson RM, Buell CR (2011) Gene coexpression network analysis as a source of functional annotation for rice genes. PloS one 6(7):e22196 https://doi.org/10.1371/journal.pone.0022196

Chin $\mathrm{CH}$, Chen SH, Wu HH, Ho CW, Ko MT, Lin CY (2014) cytoHubba: identifying hub objects and subnetworks from complex interactome. BMC systems biology 8 Suppl 4(Suppl 4):S11S11https://doi.org/10.1186/1752-0509-8-S4-S11

Cline MS, Smoot M, Cerami E, Kuchinsky A, Landys N, Workman C, Christmas R, Avila-Campilo I, Creech $M$, Gross $B(2007)$ Integration of biological networks and gene expression datausing Cytoscape. Nat Protoc (2):2366-2382

https://doi.org/10.1038/nprot.2007.324

Dardenne M (2002) Zinc and immune function. European Journal of Clinical Nutrition (56):S20-S23 https://doi.org/10.1038/sj.ejcn.1601479

Debi BR, Taketa S, Ichii M (2005) Cytokinin inhibits lateral root initiation but stimulates lateral Root elongation in rice (Oryza sativa). Journal of plant physiology 162(5):507-515 https://doi.org/10.1016/j.jplph.2004.08.007

El-Shabrawi H, Kumar B, Kaul T, Reddy MK, Singla-Pareek SL, Sopory SK (2010) Redox homeostasis, antioxidant defense, and methylglyoxal detoxification as markers for salt tolerance in Pokkali rice. Protoplasma 245(1):85-96

https://doi.org/10.1007/s00709-0100144-6

Fan X, Feng H, Tan Y, Xu Y, Miao Q, Xu G (2016) A putative 6-transmembrane nitrate transporter OsNRT1.1b plays a key role in rice under low nitrogen. Integr Plant Biol 58(6):590-599. https://doi.org/10.1111/jipb.12382

Hartwell LH, Hopfield JJ, Leibler S, Murray AW (199) From molecular to modular cell biology. Nature 402(6761):C47-52 https://doi.org/10.1038/35011540

Hu B, Wang W, Ou S, Tang J, Li H, Che R, Zhang Z, Chai X, Wang H, Wang Y, Liang C, Liu L, Piao Z, Deng Q, Deng K, Xu C, Liang Y, Zhang L, Li L, Chu C (2015) Variation in NRT1.1B contributes to nitrate-use divergence between rice subspecies. Nat Genet 47(7): 834-838 https://doi.org/10.1038/ng.3337

Huizar MI, Arena R, Laddu DR (2021) The global food syndemic: the impact of food insecurity, malnutrition and obesity on the healthspan amid the COVID-19 pandemic. Progress in cardiovascular diseases 64:105-107 https://dx.doi.org/10.1016\%2Fj.pcad.2020.07.002 
Ishimaru Y, Bashir K, Nishizawa NK (2011) Zn Uptake and Translocation in Rice Plants. Rice 4:21-27 https://doi.org/10.1007/s12284-011-9061-3

Ishimaru Y, Suzuki M, Kobayashi T, Takahashi M, Nakanishi H, Mori S, Nishizawa NK (2005) OsZIP4, a novel zinc-regulated zinc transporter in rice. Journal of Experimental Botany 56(422):3207-3214 https://doi.org/10.1093/jxb/eri317

Katiyar A, Smita S, Lenka SK, Rajwanshi R, Chinnusamy V, Bansal KC (2012) Genome-wide classification and expression analysis of MYB transcription factor families in rice and Arabidopsis. BMC genomics (1):1-9 https://doi.org/10.1186/1471-2164-13-544

Keller C, Rizwan M, Davidian JC, Pokrovsky OS, Bovet N, Chaurand P, Meunier JD (2015) Effect of silicon on wheat seedlings (Triticum turgidum L.) grown in hydroponics and exposed to 0 to $30 \mu \mathrm{M} \mathrm{Cu}$. Planta 241(4):847-860 https://doi.org/10.1007/s00425-014-2220-1

Kitomi Y, Hidemi K, Inukai Y (2011) Molecular mechanism of crown root initiation and the different mechanisms between crown root and radicle in rice. Plant signaling, behavior 6(9): 1276-1278 https://doi.org/10.4161/psb.6.9.16787

Langfelder P, Horvath S (2008) WGCNA: an R package for weighted correlation network analysis. BMC bioinformatics 9(1):1-13 https://doi.org/10.1186/1471-2105-9-559

Lee S, Chiecko JC, Kim SA, Walker EL, LeeY, Guerinot ML, An G (2009) Disruption of OsYSL15 leads to iron inefficiency in rice plants. Plant physiology 150(2):786-800

https://doi.org/10.1104/pp.109.135418

Lee S, Kim SA, Lee J, Guerinot ML, An G (2010) Zinc deficiency-inducible OsZIP8 encodes a plasma membrane-localized zinc transporter in rice. Mol Cells (29):551-558

https://doi.org/10.1007/s10059-010-0069-0

Li H, Handsaker B, Wysoker A, Fennell T, Ruan J, Homer N, Marth G, Abecasis G, Durbin R (2009) The sequence alignment/map format and SAMtools. Bioinformatics 25(16):2078-2079

https://doi.org/10.1093/bioinformatics/btp352

Li J, Han Y, Liu L, Chen Y, Du Y, Zhang J, Sun H, Zhao Q (2015) qRT9, a quantitative trait locus controlling root thickness and root length in upland rice. Journal of Experimental Botany 66(9):2723-32 https://doi.org/10.1093/jxb/erv076

Liao Y, Smyth GK, Shi W (2014) featureCounts: an efficient general purpose program for assigning sequence reads to genomic features. Bioinformatics 30(7):923-930 https://doi.org/10.1093/bioinformatics/btt656 
Lu X, Liu S, Zhi S, Chen J, Ye G (2020) Comparative transcriptome profile analysis of rice varieties with different tolerance to zinc deficiency. Plant Biol 23(2):375-390 https://doi.org/10.1111/plb.13227

Ma JF, Yamaji N, Mitani N, Xu XY, Su YH, McGrath SP, Zhao FJ (2008) Transporters of arsenite in rice and their role in arsenic accumulation in rice grain. Proceedings of the National Academy of Sciences 105(29):9931-5 https://doi.org/10.1073/pnas.0802361105

Masuda H, Ishimaru Y, Aung MS, Kobayashi T, Kakei Y, Takahashi M, Higuchi K, Nakanishi H, Nishizawa NK (2012) Iron biofortification in rice by the introduction of multiple genes involved in iron nutrition. Sci Rep 2, 543 https://doi.org/10.1038/srep00543

Maurya S, Vishwakarma AK, Dubey M, Shrivastava P, Shrivastava R, Chandel G (2018) Developing genetagged molecular marker for functional analysis of OsZIP10 metal transporter gene in rice. Indian Journal of Genetics and Plant Breeding (The) 78(2): 180-186. https://doi.org/10.5958/0975-6906.2018.00023.8

Mering VC, Huynen M, Jaeggi, D, Schmidt S, Bork P, Snel B (2003) STRING: a database of predicted functional associations between proteins. Nucleic Acids Research 31(1):258-261 https://doi.org/10.1093/nar/gkg034

Nanda AK, Pujol V, Wissuwa M (2017) Patterns of stress response and tolerance based on transcriptome profiling of rice crown tissue under zinc deficiency. Journal of Experimental Botany 68(7):1715-1729 https://doi.org/10.1093/jxb/erx039

Nanda AK, Wissuwa M (2016a) Rapid crown root development confers tolerance to zinc deficiency in rice. Frontiers in plant science 7, 428 https://doi.org/10.3389/fpls.2016.00428

Neogy A, Garg T, Kumar A, Dwivedi AK, Singh H, Singh U, Singh Z, Prasad K, Jain M, Yadav S (2019) Genome-Wide Transcript Profiling Reveals an Auxin-Responsive Transcription Factor, OsAP2/ERF-40, Promoting Rice Adventitious Root Development. Plant Cell Physiol 60(10):2343-2355 https://doi.org/10.1093/pcp/pcz132

Noctor G, Mhamdi A, Chaouch S, Han Y, Neukermans J, Marquez-Garcia B, Queval G, Foyer C H (2012) Glutathione in plants: an integrated overview. Plant Cell Environ 35(2):454-84 https://doi.org/10.1111/j.1365-3040.2011.02400.x

Nozoye T, Nagasaka S, Kobayashi T, Takahashi M, Sato Y, Sato Y, Uozumi N, Nakanishi H, Nishizawa NK (2011) Phytosiderophore efflux transporters are crucial for iron acquisition in graminaceous plants. Journal of Biological Chemistry 286(7):5446-54.

https://doi.org/10.1074/jbc.M110.18002

Ogo Y, Itai RN, Nakanishi H, Inoue H, Kobayashi T, Suzuki M, Takahashi M, Mori S, Nishizawa NK (2006) Isolation and characterization of IRO2, a novel iron-regulated bHLH transcription factor in graminaceous plants. Journal of Experimental Botany 57, 2867-78 https://doi.org/10.1093/jxb/erl054 
Ogo Y, Itai RN, Kobayashi T, Nakanishi H, Mori S, Nishizawa NK (2009) Overexpression of OsIRO2 improves both iron uptake and translocation to seeds in rice. UC Davis: Department of Plant Sciences. https://escholarship.org/uc/item/2kd7m133 .Accessed 15 Apr 2021

Ogo Y, Nakanishi IR, Nakanishi H, Kobayashi T, Takahashi M, Mori S, Nishizawa NK (2007) The rice bHLH protein OsIRO2 is an essential regulator of the genes involved in Fe uptake under Fe-deficient conditions. Plant journal 51(3):366-377

https://doi.org/10.1111/j.1365-313X.2007.03149.x

Olsen LI, Palmgren MG (2014) Many rivers to cross: the journey of zinc from soil to seed. Frontiers in Plant Science 5, 30 https://doi.org/10.3389/fpls.2014.00030

Pérez-Rodríguez P, Riano-Pachon DM, Corrêa LG, Rensing SA, Kersten B, Mueller-Roeber B (2010) PInTFDB: updated content and new features of the plant transcription factor database. Nucleic acids research 38(suppl_1):D822-7 https://doi.org/10.1093/nar/gkp805

Ptashnyk M, Roose T, Jones DL, Kirk G (2011) Enhanced zinc uptake by rice through phytosiderophore secretion: a modelling study. Plant Cell Environ 34(12):2038-2046

https://doi.org/10.1111/j.1365-3040.2011.02401.x

Ramírez-González RH, Borrill P, Lang D, Harrington SA, Brinton J, Venturini L, Davey M, Jacobs J, Van Ex F, Pasha A (2018) The transcriptional landscape of polyploid wheat. Science 361(6403) https://doi.org/10.1126/science.aar6089

Sasaki A, Yamaji N, Ma JF (2014) Overexpression of OsHMA3 enhances Cd tolerance and expression of $\mathrm{Zn}$ transporter genes in rice. Journal of experimental botany 65(20):6013-6021 https://doi.org/10.1093/jxb/eru340

Seneviratne G, Jayasinghearachchi HS (2003) Mycelial colonization by bradyrhizobia and azorhizobia. Journal of biosciences 28(2):243-247 https://doi.org/10.1007/BF02706224

Sharma A, Shahzad B, Rehman A, Bhardwaj R, Landi M, Zheng B (2019) Response of phenylpropanoid pathway and the role of polyphenols in plants under abiotic stress. Molecules 24(13):2452 https://doi.org/10.3390/molecules24132452

Sinclair SA, Krämer U (2012) The zinc homeostasis network of land plants. Biochimica et Biophysica Acta -Molecular Cell Research 1823(9):1553-1567

https://doi.org/10.1016/j.bbamcr.2012.05.016

Singh SP, Gruissem W, Bhullar NK (2017) Single genetic locus improvement of iron, zinc and $\beta$-carotene content in rice grains. Scientific Reports 7(1):6883 
https://doi.org/10.1038/s41598-017-07198-5

Sichul L, Jeff CC, Sun AK, Elsbeth LW, Youngsook L, Mary LG, Gynheung A (2009) Disruption of OsYSL15 leads to iron inefficiency in rice plants. Plant physiology 150(2):786-800 https://doi.org/10.1104/pp.109.135418

Song A, Li P, Fan F, Li Z, Liang Y (2014) The effect of silicon on photosynthesis and expression of its relevant genes in rice (Oryza sativa L.) under high-zinc stress. PLoS One 9(11):e113782 https://doi.org/10.1371/journal.pone.0113782

Suzuki M, Bashir K, Inoue H, Takahashi M, Nakanishi H, Nishizawa NK (2012) Accumulation of starch in Zn-deficient rice. Rice 5(1):1-8 https://doi.org/10.1186/1939-8433-5-9

Takahashi R, Bashir K, Ishimaru Y, Nishizawa NK, Nakanishi H (2012) The role of heavy-metal ATPases, HMAs, in zinc and cadmium transport in rice. Plant signaling \&amp; behavior 7(12):1605-1607 https://doi.org/10.4161/psb.22454

Tan M, Cheng D, Yang Y, Zhang G, Qin M, Chen J, Chen Y, Jiang M (2017) Co-expression network analysis of the transcriptomes of rice roots exposed to various cadmium stresses reveals universal cadmiumresponsive genes. BMC Plant Biology 17(1):194

https://doi.org/10.1186/s12870-017-1143-y

Tripathi P, Tripathi RD, Singh RP, Dwivedi S, Goutam D, Shri M, Trivedi PK, Chakrabarty D (2013) Silicon mediates arsenic tolerance in rice (Oryza sativa L.) through lowering of arsenic uptake and improved antioxidant defence system. Ecological engineering 52:96-103

https://doi.org/10.1016/j.ecoleng.2012.12.057

Van DSD, Bhullar NK, De SH, Gruissem W, MacKenzie D, Pfeiffer W, Qaim M, Slamet-Loedin I, Strobbe S, Tohme J, Trijatmiko KR, Vanderschuren H, Van MM, Zhang C, Bouis H (2020) Multiplying the efficiency and impact of biofortification through metabolic engineering. Nature Communications 11(1):5203 https://doi.org/10.1038/s41467-020-19020-4

Walley JW, Sartor RC, Shen Z, Schmitz RJ, Wu KJ, Urich MA, Nery JR, Smith LG, Schnable JC, Ecker JR (2016) Integration of omic networks in a developmental atlas of maize. Science 353(6301):814-818 https://doi.org/10.1126/science.aag1125

Wang W, Ye J, Ma Y, Wang T, Shou H, Zheng L (2020) OsIRO3 Plays an Essential Role in Iron Deficiency Responses and Regulates Iron Homeostasis in Rice. Plants (Basel) 9(9):1095

https://doi.org/10.3390/plants9091095

Widodo B, Broadley MR, Rose T, Frei M, Pariasca-Tanaka J, Yoshihashi T, Thomson M, Hammond JP, Aprile A, Close TJ, Ismail AM, Wissuwa M (2010) Response to zinc deficiency of two rice lines with 
contrasting tolerance is determined by root growth maintenance and organic acid exudation rates, and not by zinc-transporter activity. New Phytol 186(2):400-414 https://doi.org/10.1111/j.1469-

8137.2009.03177.x

Yamaji N, Sakurai G, Mitani-Ueno N, Ma JF (2015) Orchestration of three transporters and distinct vascular structures in node for intervascular transfer of silicon in rice. Proc Natl Acad Sci 112(36):1140111406 https://doi.org/10.1073/pnas.1508987112

Yamaji Ni, Xia J, Mitani-Ueno N, Yokosho K, Ma JF (2013) Preferential delivery of zinc to developing tissues in rice is mediated by P-type heavy metal ATPase OsHMA2. Plant physiology 162(2):927-939 https://doi.org/10.1104/pp.113.216564

Yang Z, Wu Y, Li Y, Ling HQ, Chu C (2009) OsMT1a, a type 1 metallothionein, plays the pivotal role in zinc homeostasis and drought tolerance in rice. Plant molecular biology 70(1-2): 219-229 https://doi.org/10.1007/s11103-009-9466-1

Zeng $\mathrm{H}$, Zhang $\mathrm{X}$, Ding M, Zhu Y (2019b) Integrated analyses of miRNAome and transcriptome reveal zinc deficiency responses in rice seedlings. BMC Plant Biol 19(1):585 https://doi.org/10.1186/s12870-0192203-2

Zhang B, Horvath S (2005) A general framework for weighted gene co-expression network analysis. Stat Appl Genet Mol Biol 4(1): Article17 https://doi.org/10.2202/1544-6115.1128

Zhang L, Hu B, Deng K, Gao X, Sun G, Zhang Z, Li P, Wang W, Li H, Zhang Z, Fu Z, Yang J, Gao S, Li L, Yu F, Li Y, Ling H, Chu C (NRT1.1B) improves selenium concentrations in rice grains by facilitating selenomethinone translocation. Plant Biotechnol 17(6):1058-1068 https://doi.org/10.1111/pbi.13037

Zhang Y, Yu C, Lin J, Liu J, Liu B, Wang J, Huang A, Li H, Zhao T (2017) OsMPH1 regulates plant height and improves grain yield in rice. PLoS One 12(7): e0180825

https://doi.org/10.1371/journal.pone.0180825

Zhao W, Langfelder P, Fuller T, Dong J, Li A, Hovarth S (2010) Weighted gene coexpression network analysis: state of the art. Journal of biopharmaceutical statistics 20(2):281-300 https://doi.org/10.1080/10543400903572753

Zheng L, Ying Y, Wang L, Wang F, Whelan J, Shou H (2010) Identification of a novel iron regulated basic helix-loop-helix protein involved in Fe homeostasis in Oryza sativa. BMC Plant Biol 10:166 https://doi.org/10.1186/1471-2229-10-166

Zheng Y, Jiao C, Sun H, Rosli HG, Pombo MA, Zhang P, Banf M, Dai X, Martin GB, Giovannoni J (2016) ITAK: a program for genome-wide prediction and classification of plant transcription factors, transcriptional regulators, and protein kinases. Molecular plant 9(12):1667-1670 https://doi.org/10.1016/j.molp.2016.09.014 
Figures

Fig. 1

(a)

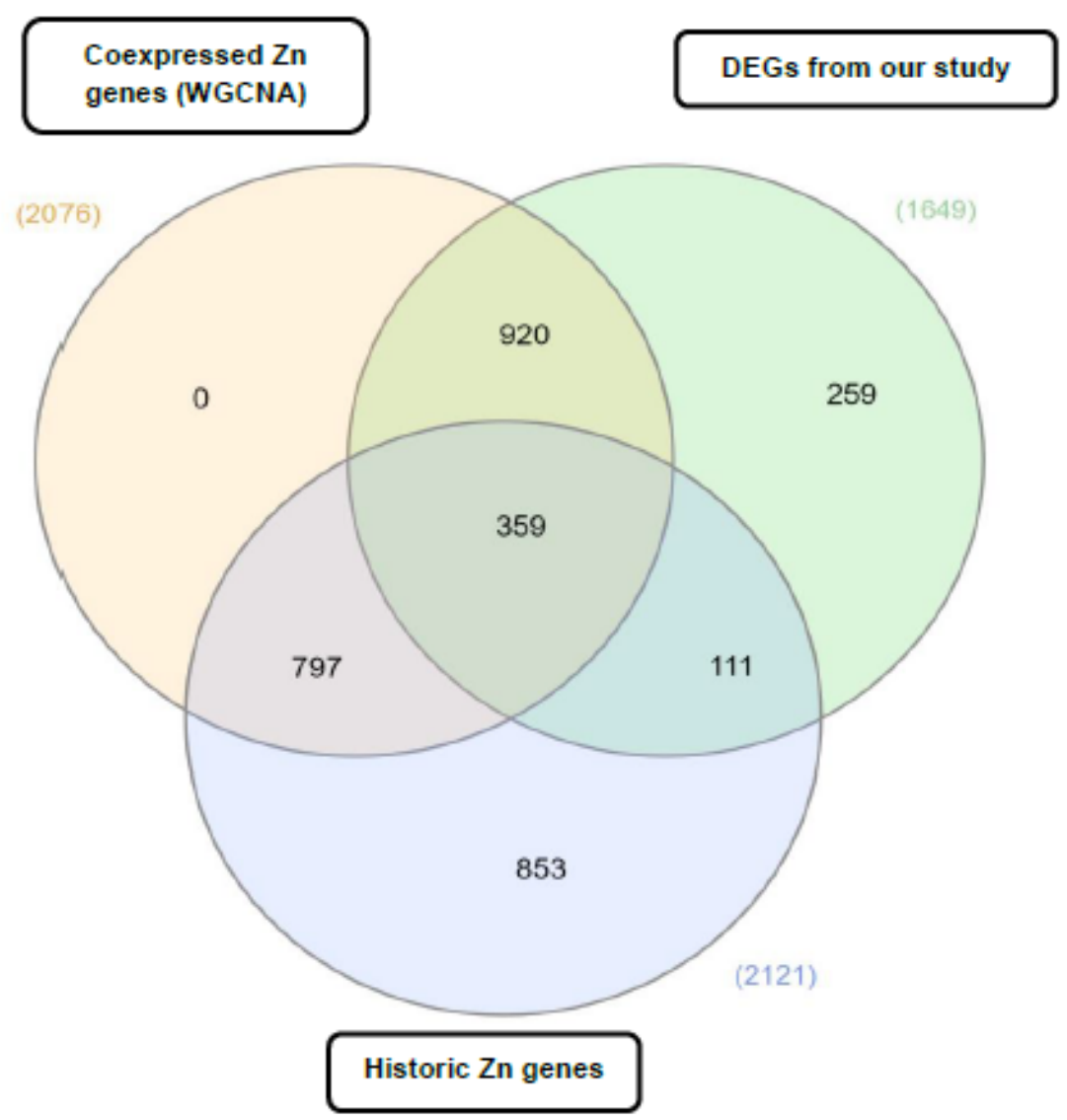

(b)

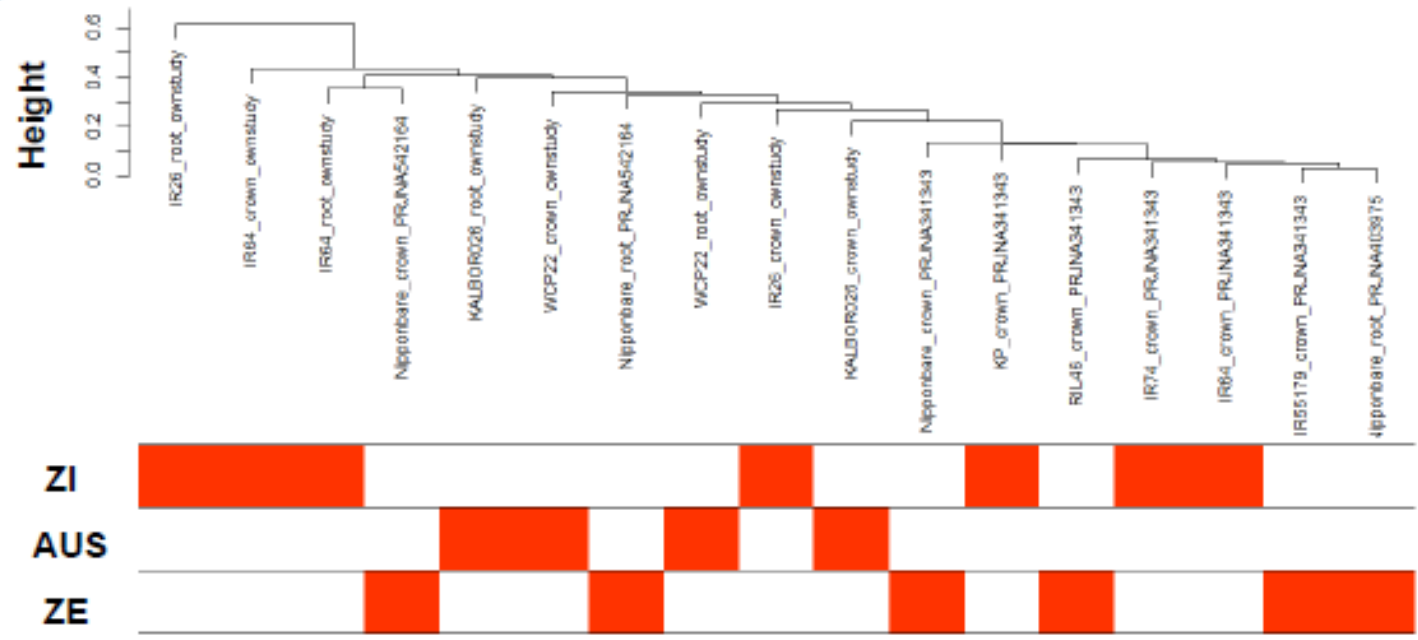

\section{Figure 1}

Genes and sample genotypes used for WGNA. a A three-level venn diragram illustrating overlapping genes between DEGs from this study, coexpressed genes and reported Zn genes from GWAS, qtl mapping, transgenic, miRNA and gene transcriptomic studies. b Sample dendrogram and classification 
of 17 used samples into different ZUE categories. Height represents the average Euclidean distance between samples computed with hclust() function. A heat map of ZUE categories across all genotypes is illustrated underneath the sample dendrogram. Genotypes from the same category of ZUE are represented by red-colored bars. ZUE levels of AUS genotypes are still unknown. Therefore, no specific categories was attributed to AUS genotypes.

Fig. 2

(a)

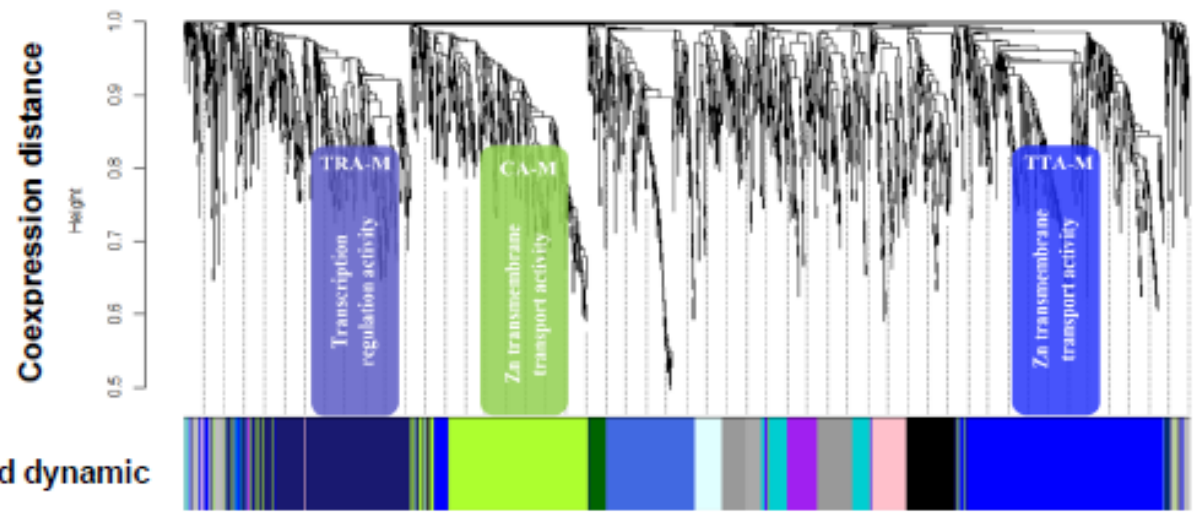

(b)

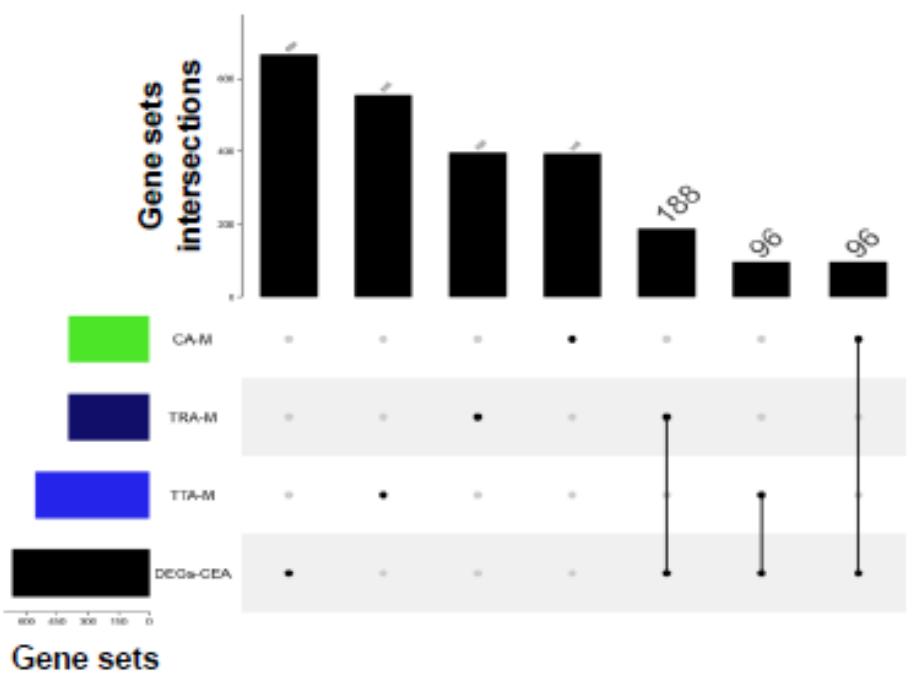

(c)

\begin{tabular}{|c|c|c|c|}
\hline \multicolumn{4}{|c|}{ TTA-M } \\
\hline SAMPLES & GO TERM/KEGG ID & GO TERM/KEGG ID DESCRIPTION & FDR \\
\hline \multirow{3}{*}{ ROOT_KALBOR026 } & GO:0015250 & \multirow{3}{*}{$\begin{array}{l}\text { Water channel activity } \\
\text { transmembrase transport } \\
\text { Glutathione metabolism }\end{array}$} & 0.01 \\
\hline & GO:0055085 & & 0.0216 \\
\hline & $\operatorname{map} 00480$ & & 0.0074 \\
\hline \multirow{2}{*}{ ROOT UCP122 } & map00480 & Glutathione metabolism & 0.00084 \\
\hline & \multicolumn{3}{|c|}{ TRA-M } \\
\hline \multirow{3}{*}{ ROOT_KALEORO26 } & GO:0003700 & DNA-binding transcription factor activity & 0.0021 \\
\hline & GO:0043565 & Sequence-specific DNA binding & 0.0111 \\
\hline & GO:0010200 & Response to chitin & 0.02 \\
\hline \multirow{4}{*}{ ROOT_LCP122 } & GO:0003700 & DNA-binding transcription factor activity & 0.000182 \\
\hline & GO:0030244 & Cellulose biosynthetic process & 0.0128 \\
\hline & GO:0050896 & Response to stimulus & 0.03 \\
\hline & GO:0043565 & Sequence-specific DNA binding & 0.03 \\
\hline \multicolumn{4}{|c|}{ CA-M } \\
\hline \multirow{4}{*}{ ROOT_IR26 } & mapo1 110 & Biosynthesis of secondary metabolites & 0.0191 \\
\hline & map00130 & Ubiquinone and other terpenoid-quinone biosynthesis & 0.0275 \\
\hline & map 00360 & Phenylalanine metabolism & 0.0275 \\
\hline & mary00940 & Phenylpropanoid biosynthesis & 0.0275 \\
\hline
\end{tabular}

Figure 2 
Preserved genes and molecular pathways in ZDR modules. a Hierarchical dendrogram of coexpressed genes/nodes and visualization of ZDR modules. Coexpression distance is equivalent to the topological dissimilarity of nodes (1-TOM). Modules were constructed based on dynamic tree cutting method (see materials and methods for details). b Upset graph representing the number of coexpressed DEGs in ZDR modules. Numbers with larger font size at the the top of black bar graphs in the upper pannel represent intersecting genes between DEGs and ZDR modules. c Functional annotations of the preserved DEGs in each ZDR module. A statistical threshold (FDR $<0.05)$ was applied to define the significantly affected GO or KEGG pathways. 
Fig. 3

Network legend

Expressed in this study
$\begin{aligned} & \text { Expressed in this } \\ & \text { study or previous studies }\end{aligned}$

(a)

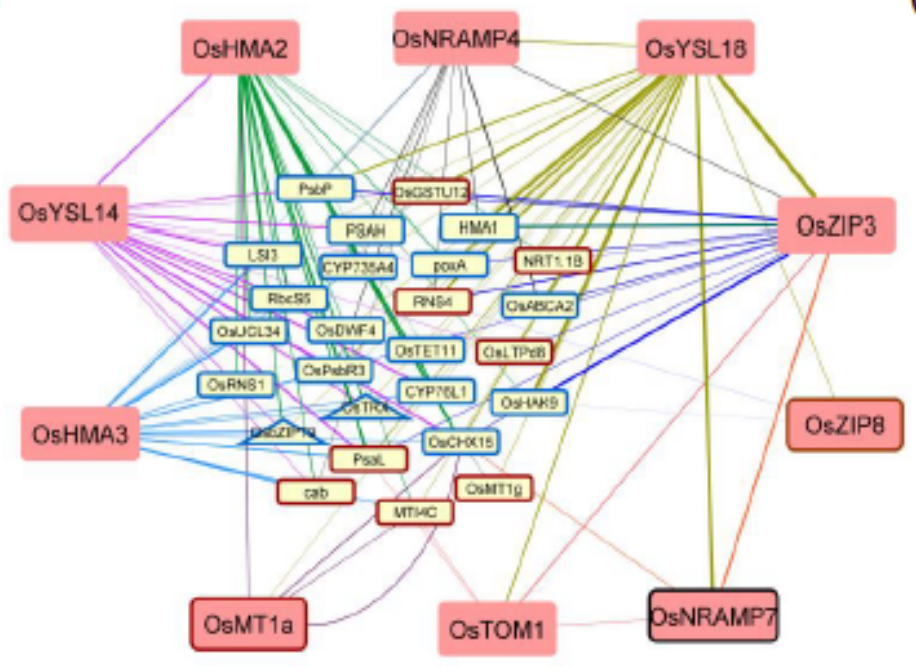

(b)

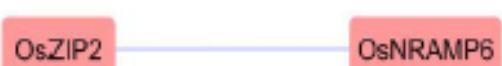

(c)

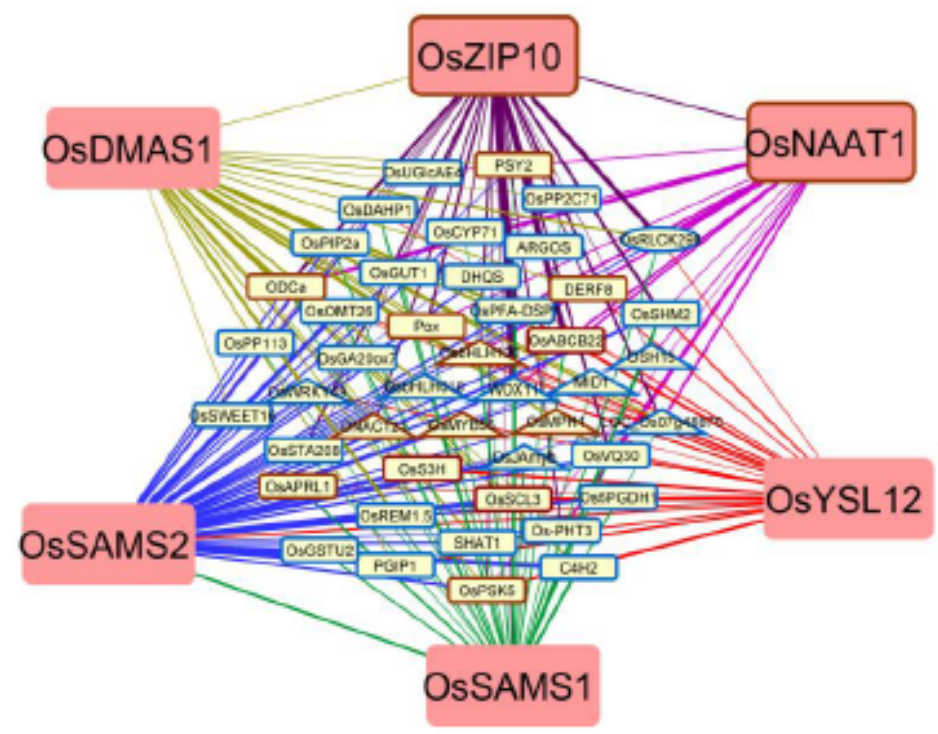

\section{Figure 3}

Visualization of gene-gene interaction networks of ZDR modules. a TTA-M module. b TRA-M module. c CA-M module. Edges represent interactions. Edges from the interactions between the well-known ZDR genes (bigger nodes and font size) and other genes are represented with different colored lines (edges). Genes at the center of each module (hub genes) interact with at least three well-known ZDR genes. The 
thicker the edge, the stronger is the degree of connections between the nodes it links. Bordered nodes correspond to DEGs from this study. A more detailed network legend is provided at the top of the panel.

\section{Supplementary Files}

This is a list of supplementary files associated with this preprint. Click to download.

- CaptionsforSupplementalTablesandFigures.docx

- Additionalfile1.xlsx

- Additionalfile10.xlsx

- Additionalfile3.xlsx

- Additionalfile11.xlsx

- Additionalfile12.xlsx

- Additionalfile13.xlsx

- Additionalfile14.xIsx

- Additionalfile15.xIsx

- Additionalfile16.xlsx

- Additionalfile17.xlsx

- Additionalfile18.xlsx

- Additionalfile19.xlsx

- Additionalfile2.xlsx

- Additionalfile20.xlsx

- Additionalfile21.xlsx

- Additionalfile22.xIsx

- Additionalfile23.xlsx

- Additionalfile24.xlsx

- Additionalfile25.xlsx

- Additionalfile26.xlsx

- Additionalfile27.xlsx

- Additionalfile28.xlsx

- Additionalfile29.xlsx

- Additionalfile30.xlsx

- Additionalfile31.xlsx

- Additionalfile32.xlsx

- Additionalfile33.xlsx 
- Additionalfile34.xlsx

- Additionalfile35.xlsx

- Additionalfile36.xIsx

- Additionalfile37.xIsx

- Additionalfile38.docx

- Additionalfile4.xlsx

- Additionalfile5.xIsx

- Additionalfile6.xlsx

- Additionalfile7.xlsx

- Additionalfile8.xIsx

- Additionalfile9.xlsx 